\title{
The Influence of Continuous-Wave and Modulated Interference on Incoherent Spectral Codes
}

\author{
B. Huiszoon (1), T. Spuesens (2), E. Tangdiongga (3), and A.M.J. Koonen (3) \\ 1: Was with COBRA Institute, now with Networking Research Group, Universidad Autónoma de Madrid, 28049 Madrid, Spain. \\ 2: Was with COBRA Institute, now with INTEC, Ghent University, Sint-Pietersnieuwstraat 41, 9000 Ghent, Belgium. \\ 3: COBRA Institute, Eindhoven University of Technology, PO BOX 513, 5600MB Eindhoven, The Netherlands. \\ bas.huiszoon@uam.es, spuesens@intec.ugent.be, \{e.tangdiongga,a.m.j.koonen\}@tue.nl
}

\begin{abstract}
We show, for the first time, simulation and experimental results on the resilience of incoherent spectral codes to continuous-wave and amplitude-modulated interference which may be applied to opticalwireless communication and optical packet switching. (C) 2009 Optical Society of America

OCIS codes: (060.2330) Fiber optics communications; (060.4785) Optical security and encryption
\end{abstract}

\begin{abstract}
1. Introduction
Optical code division multiple access (OCDMA) resembles spread spectrum CDMA techniques employed in the radio frequency (RF) domain and may provide physical layer security in terms of confidentiality, integrity and availability [1]. The first two forms of security are important in case that an eavesdropper is present in the network while the latter should always be provided because it is simpler to launch an interference signal to jam the system. Typically, an RF CDMA system tolerates a fair amount of narrowband interference, and therefore a similar performance may be expected for optical CDMA. Essentially, the demonstrated OCDMA systems may be classified according to their coding principle (incoherent or coherent) and their coding domain (time, wavelength, or time and wavelength). To the best of the authors' knowledge, the influence of an in-band interferer on the transmission performance has only been studied for a coherent wavelength-domain OCDMA system [2,3]. In this work however, an incoherent wavelength-domain OCDMA system is considered. For the first time, the system's tolerance to an inband interferer is analyzed considering continuous-wave (CW) and amplitude-modulated (AM) wavelength channels. The paper is organized as follows. The operational principle and the setup are introduced in section 2 after which section 3 and 4 show simulation and experimental results. The paper concludes with a discussion in section 5 .
\end{abstract}

\section{Operational principle and transmission setup}

Incoherent wavelength-domain optical codes are amplitude distributions in the spectral domain, e.g. A(f), and correspond to the transfer function of an optical filter which filters a flat broadband optical signal. Such filters are typically placed at the transmitter and at the receiver, and as such represent the encoder (ENC) and decoder (DEC). In this work, the ENC and DEC are implemented by Mach-Zehnder (MZ) interferometer based devices which naturally generate two complementary filter patterns, i.e. A(f) and A_(f), used in spectral shift keying (SSK). SSK transmits A(f) as " 1 " and A_(f) as " 0 " such that the input data is transformed into a sequence of complementary filter patterns. As the en/decoding operation is based on optical intensity, an in-band optical channel may easily cause significant interference and, as a consequence, result in transmission errors or even system shutdown.

In principle, if the transfer function at the DEC matches with the transmitted spectrum, an optical power imbalance appears at the DEC outputs because $A(f)$ passes through the $A(f)$ port while a significant amount is blocked by the A_(f) port, and vice versa. The codes are designed in such a way that a mismatch results in average optical power at the DEC outputs. The optical powers are subtracted by a balanced photo diode (PD), and therefore result in either bipolar or close-to-zero electrical signals. If an optical interferer is well-positioned with respect to the spectral code its effect on the detected signal is minimized while if it is badly positioned it may cause significant interference at either the "1" or the "0" level. The transmission setup is depicted in Fig. 1. The output of a broadband superluminescent light emitting diode (SLED) is filtered by a band-pass filter (BPF) of which the $-3 \mathrm{~dB}$ bandwidth equals to one period of the free spectral range of the MZ-based tree ENC [4]. Tunable phase shifters are used in the ENC to generate multiple sets of orthogonal spectral codes. The optical stream is then launched into a $3 \mathrm{~dB}$ coupler after passing through a variable optical attenuator (ATT) and an amplifier (AMP), including an amplified spontaneous emission BPF, to compensate the losses of coding and modulation. An interference signal is added and, depending on which scenario, either a CW distributed feedback (DFB) laser or the AM signal is picked via setting 1 or 2. Note that a back-to-back configuration is used here to isolate the effects of interference. At the receiver-side (setting 1), the optical stream is coupled into a cascade DEC after which a balanced PD with a trans-impedance amplifier (TIA) detects the optical intensity. The electrical signal is amplified, and filtered by a low-pass filter (LPF). Regarding setting 2, a second $3 \mathrm{~dB}$ coupler is used such that the optical networking unit and a directdetection (DD) receiver recover the encoded and AM data, respectively. The DD receiver has a direct current (DC) 


\section{OWR1.pdf}

block to cancel the DC offset inferred by SSK. Average optical power levels are shown in the table-inset of Fig. 1. The simulated balanced PD is constructed by subtracting the output of two standard PIN PDs which have a sensitivity of $-20 \mathrm{dBm}$. The device used in the experiments has a much lower value, and therefore the large difference appears at measurement point $\mathbf{D}$ because simulations and experiments are done at the sensitivity-limit.

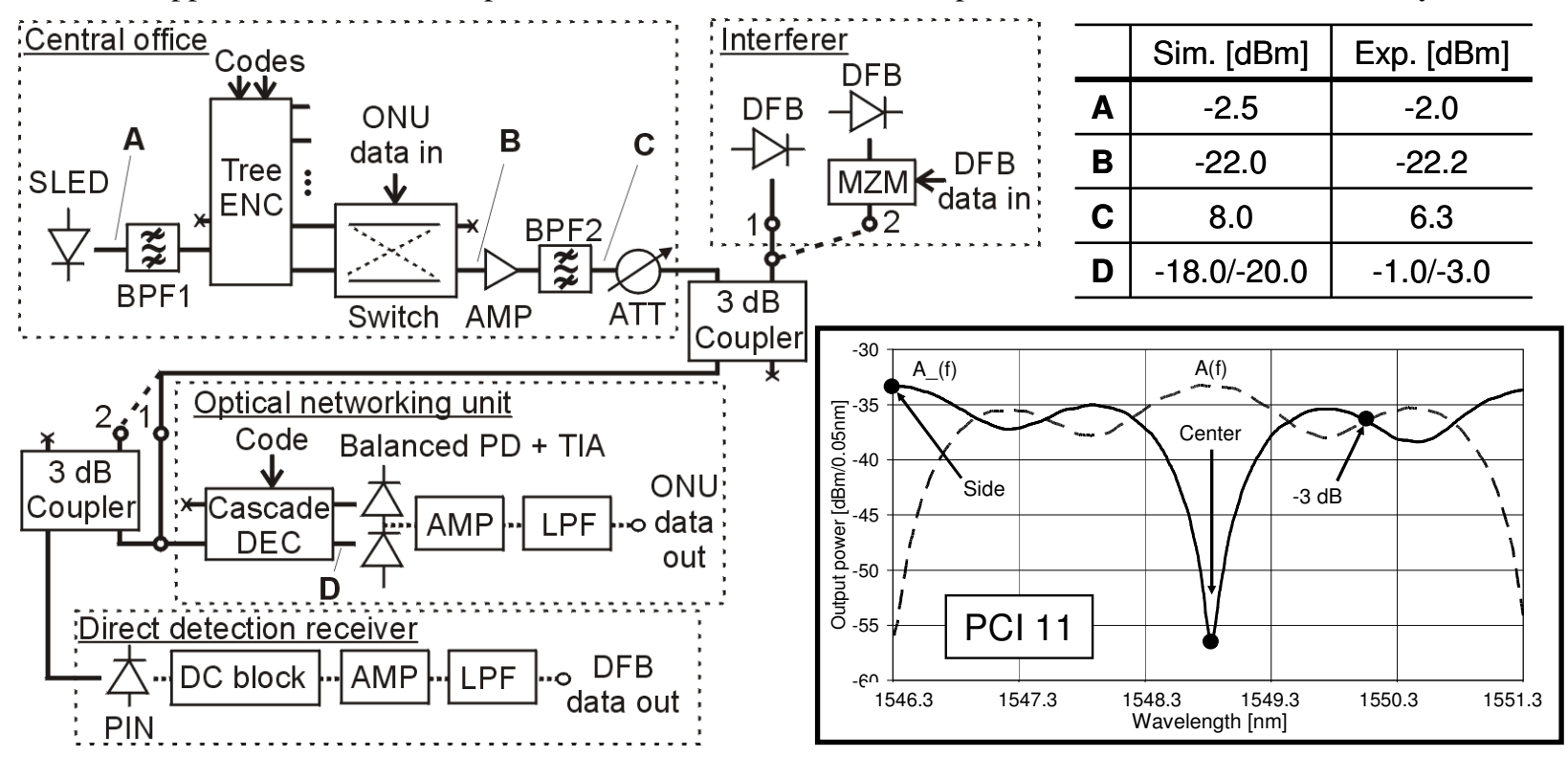

Fig. 1. Spectral code transmission system with continuous-wave or modulated interferer (inset: measured code [4] with interference positions)

3. Simulation results

The setup shown in Fig. 1 is simulated in [5] and its performance is analyzed via the quality of the eye diagrams at the receiver by means of the eye-opening factor (EOF). The EOF is a statistical metric defined in the simulation software and may be used to express penalties at increasing interference powers. All simulations have been done with the spectral codes denoted as "PCI 11" in [4] in order to allow the results to be verified by experiments. More complicated codes have been used in [6] using a similar setup. We have benchmarked the simulated system and error-free operation or severely degraded performance is found for EOF values above 0.80 or below 0.65 , respectively. A measured PCI 11 (a complementary set of spectral codes; $5 \mathrm{~nm}$ wide) is shown in the inset of Fig. 1 with three positions indicated namely "Center", "Side" and "-3 dB" to which the wavelength of the DFB lasers are tuned. The first two positions represent the worst-case scenario because the interferer is placed on a pass band of either A(f) or A_(f). The "-3 dB" position represents the best-case because the interferer power is equally spread and, via balanced detection, should be reduced to a small contribution. In the simulation the DFB's output power is varied from -20 to $+10 \mathrm{dBm}$ in steps of $1 \mathrm{~dB}$ considering two cases: firstly for optical code transmission at the receiver's sensitivity limit by adjusting the ATT value, and secondly after lowering the ATT with 10 dB. Relatively, both cases should give similar results. At each step the EOF value is calculated after receiving 64 bits at $1 \mathrm{Gbit} / \mathrm{s}$ using a standard pseudo-random bit sequence (PRBS) generator in [5]. A higher number of bits should be simulated in order to obtain more reliable results, however, limitations in computer processing power did not allow this here. The interference-to-code-ratio (ICR) is also determined which refers to the difference between the average power levels of the interferer and the spectral codes at the read-out of an optical spectrum analyzer.

The results are shown for a CW and AM interferer in Fig. 2(a) and (b) which confirm that the position of the interferer has a distinguishable impact on the transmission performance at higher ICR values. The positions "Side" and "Center" should give the same results while Fig. 2(a) indicates that "Side" has a slightly better performance. Most probably this is because of a tuning error in the DFB's wavelength. Fig. 2(a) and (b) indicate that the optical code transmission system may tolerate an ICR of about $15 \mathrm{~dB}$ in case of a CW and $4 \mathrm{~dB}$ for an AM interferer regardless its position in the optical code. The code is more tolerant to $\mathrm{CW}$ interference because of the time-varying intensity variations added by the AM channel to the " 1 " and " 0 " signal levels which cause a stronger closure of the eye diagrams. Optimizing the position of the interferer gives at least a $5 \mathrm{~dB}$ gain in ICR. In that case, simultaneous data transmission using an optically encoded channel and an AM data channel could be feasible. With respect to the AM data channel performance, it does not matter where the channel is placed in the spectral code because in all cases the spectral code only adds a small constant or slightly varying DC offset. It is therefore the ICR threshold value that is relevant in this case, that is, the signal power at which the AM channel becomes dominant. 


\section{OWR1.pdf}

\section{Experimental results}

The setup depicted in Fig. 1 is realized in the lab for bit error rate (BER) measurements in order to verify the performance. Only the $\mathrm{CW}$ interference could be implemented (setting 1) and the system is operated at the error-free limit $(\mathrm{ATT}=0 \mathrm{~dB})$ before the interferer is added. In the experiment, the DFB's output power is varied from -4 to +4 $\mathrm{dBm}$ in steps of $1 \mathrm{~dB}$ and the BER is measured at each step for the "Center" and "- $3 \mathrm{~dB}$ " positions for a $1.25 \mathrm{Gbit} / \mathrm{s}$ $2^{7}-1$ PRBS and $100 \mathrm{~s}$ time gating. The "Side" position did not produce accurate results, and therefore these are not shown here. The measured BER curves and eye diagrams are depicted in Fig. 3(a) and (b). The results in Fig. 3(a) indicate that the experimental system has similar trends as in Fig. 2(a), however, at lower ICR values. Already at ICR $=0 \mathrm{~dB}$ the interferer influences the performance. Fig. 3(b) shows severe interference on the +1 level if the AM channel is positioned in the "Center". The interference is spread on both levels if it is placed on the "- $3 \mathrm{~dB}$ " position.

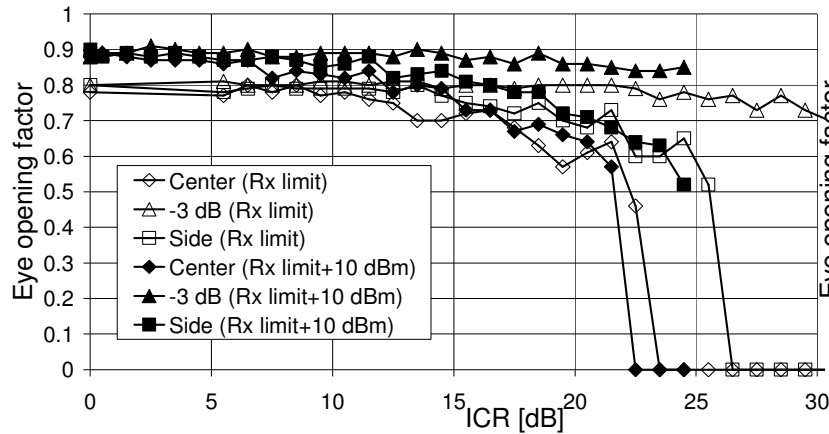

(a) Continuous-wave interferer

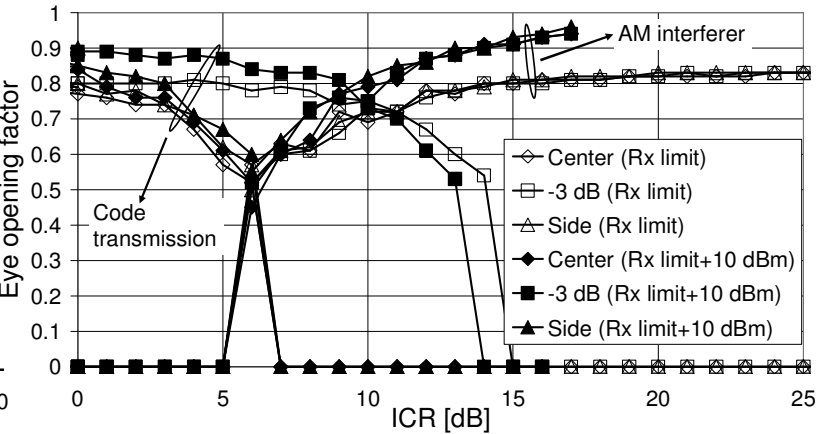

(b) Amplitude-modulated interferer

Fig. 2. Simulated interference-to-code ratio (ICR) versus eye opening factor for spectral code transmission with interference

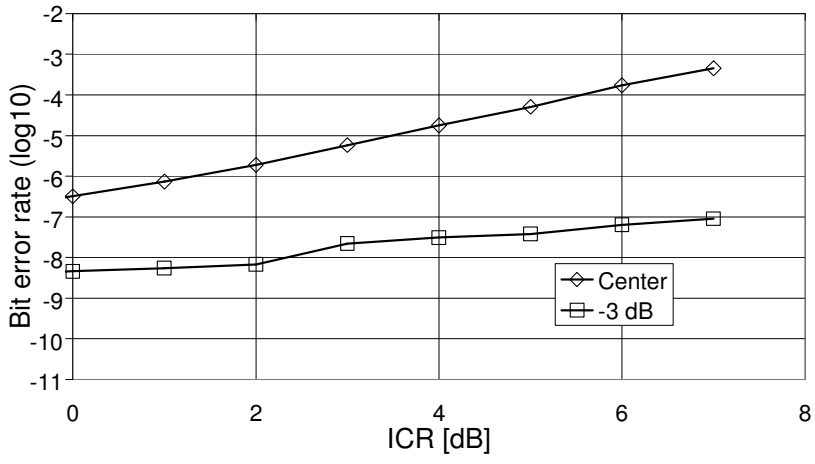

(a) Interference-to-code ratio (ICR) versus bit error rate

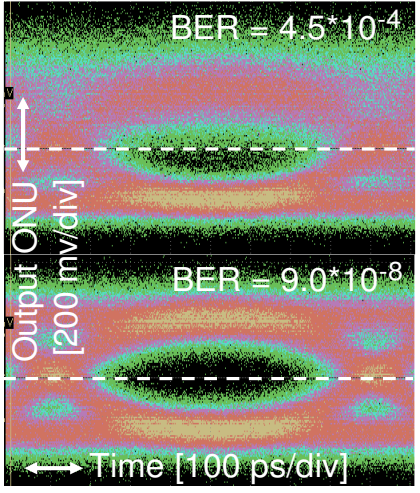

(b) Eye diagrams, ICR=7 dB; upper: "Center", lower: “-3 dB"

Fig. 3. Measured transmission performance of spectral code transmission with a continuous-wave interferer

\section{Discussion}

This work shows results of a first study on the resilience of an incoherent spectral code transmission system to inband CW and modulated optical channels via preliminary simulations and experiments. Essentially, the position of the interferer with respect to the spectral code determines the amount of interference that may be tolerated for errorfree operation. A wider spectral code increases the resilience to an interfering channel [6]. In-band multiplexing of a spectrally encoded channel and a modulated wavelength channel is found feasible in the simulations which may be applied to other applications than solely data transmission such as radio-over-fiber (RoF) and optical packet switching. Regarding the former, [7] exploited the resilience to multiplex RoF signals generated by a harmonics generating technique, i.e. optical frequency multiplication, with spectral code transmission to remotely modulate wireless channels. Regarding the latter, a spectral code may be used as an in-band label to route data packets similar to [8] which uses static in-band wavelength channels in $160 \mathrm{Gbit} / \mathrm{s}$ optical packets.

\section{References}

[1] T.H. Shake, IEEE/OSA J. Lightw. Technol., 23(2), pp. 655, 2005. [5] VPI TransmissionMaker homepage: http://www.vpiphotonics.com/.

[2] S. Shen and A. Weiner, Photon. Technol. Lett., 13(1), pp. 82, 2001. [6] B. Huiszoon, Ph.D. dissertation, Eindhoven Univ. of Technol., 2008.

[3] P.-H. Chang and S.-H. Zhou, in Proc. OFC 2004, paper FG6, 2004. [7] T. Spuesens, et al., in Proc. ECOC 2008, paper P.6.23, 2008.

[4] B. Huiszoon, et al., in Proc. OFC 2008, paper OMR4, USA, 2008. [8] N. Calabretta, et al., in Proc. OFC 2008, paper PDP33, USA, 2008.

The Dutch Ministry of Economic Affairs, Netherlands Organization for Scientific Research (NWO) and Technology Foundation STW are acknowledged for funding via the NRC Photonics Research grant and the Valorisation Grant I. 